\title{
Effects of extensor and flexor group I afferent volleys on the excitability of individual soleus motoneurones in man
}

\author{
PETER A SHBY A N D KE I T H A B E L L E \\ From Toronto Western Hospital, Toronto, Canada
}

SUMMARY The contour of the postsynaptic potential (PSP) produced in a neurone by an afferent volley can be derived from the contour of the post-stimulus time histogram (PSTH) of that neurone when it is discharging rhythmically. In the present study the PSTH of the firing of individual soleus motor units after stimulation of the popliteal or peroneal nerve was used to explore the effects of extensor and flexor group I afferent volleys on the excitability of single soleus motoneurones in man. Extensor group I volleys resulted in an early peak of increased impulse density in the PSTH of $75 \%$ of soleus motoneurones. The latency suggests an analogy with the Ia EPSP. The mean duration of the peak of increased impulse density, equivalent to the rise time of the EPSP, was $3.6 \mathrm{~ms}$. Flexor group I volleys result in a period of reduced impulse density in the PSTH of five out of nine soleus motoneurones. The latency suggests an analogy with the Ia IPSP. We conclude that this method could be used to explore the afferent connections to single motoneurones in man and to derive some of the characteristics of the postsynaptic potentials from a variety of afferent nerve fibres in single human motoneurones.

The ability to record excitatory postsynaptic potentials (EPSP) and inhibitory postsynaptic potentials (IPSP) intracellularly from neurones (Brock et al., 1952) permitted the systematic exploration of the connections to spinal motoneurones in the experimental animal. Similar information can be obtained in man only indirectly. For example, afferent connections to human motoneurones can be deduced from the way in which the monosynaptic reflex (Táboríková and Sax, 1969; Gassel and Ott, 1970; Tanaka, 1972) or ongoing electromyographic activity (Gassel and Ott, 1970; Caccia et al., 1973; Bouaziz et al., 1975) is modulated by an afferent volley or from the electromyographic activity evoked by an afferent volley (Shahani, 1970). These methods provide information only about overall responses of large populations of motoneurones and lack the precision provided by the intracellular recordings which can be carried out in animals.

Address for correspondence and reprint requests: Dr Peter Ashby, Toronto Western Hospital, 399 Bathurst Street, Toronto, Ontario M5T 2S3, Canada.

Accepted 9 May 1977
This paper describes a method that may be used to infer PSPs in single motoneurones in man. Some preliminary results concerning the effects of extensor and flexor group I volleys on single soleus motoneurones are reported.

\section{Methods}

Studies were performed on seven normal subjects aged 25-42 years (mean $31 \mathrm{yr}$ ).

The subjects lay prone with the foot resting on a foot plate which held the ankle at approximately $90^{\circ}$. Surface electrodes were placed over the soleus muscle to record the muscle compound action potential. Square wave electric stimuli (100-500 microseconds duration) were delivered to the popliteal nerve in the popliteal fossa. The position of the bipolar stimulating electrode was adjusted to obtain a reflex response in soleus (the $\mathrm{H}$ reflex) at the lowest threshold and then immobilised with a rubber strap. The latency to the onset of the maximum $\mathrm{H}$ reflex was recorded. The stimulus current was then reduced until the $\mathrm{H}$ reflex no longer occurred with every stimulus al- 
though a small response might reappear rarely as a result of spontaneous fluctuations in central excitability. This stimulus level, which was intended to produce a group I volley insufficient in itself to bring motoneurones to threshold, was designated 'group I threshold'. A second bipolar stimulating electrode was placed over the peroneal nerve at the head of the fibula and the position adjusted so that a contraction of the tibialis anterior was obtained with the lowest threshold. The stimulus current was then adjusted to produce a definite, but submaximal, contraction of the anterior compartment muscles. This stimulus level, which was intended to produce a definite group I volley, was designated 'alpha threshold'. It is recognised that local cutaneous afferent nerve fibres would be excited in both instances.

The action potentials of voluntarily activated soleus motor units were led off with a concentric needle electrode (Disa 13650, length $30 \mathrm{~mm}$, crosssection $0.65 \mathrm{~mm}^{2}$, electrode surface $0.07 \mathrm{~mm}^{2}$ ) which was inserted into the soleus $10-20 \mathrm{~mm}$ below the junction of the heads of the gastrocnemius (Fig. 1). The signals were amplified ( 0.2 to $1 \mathrm{mV}=1$ volt) and displayed using standard electromyographic apparatus (Tektronix 2A61 amplifier with band pass between $40 \mathrm{~Hz}$ and 10 $\mathrm{kHz}$; Tektronix 564 storage oscilloscope) and passed through an audioamplifier to a loudspeaker. The subject was provided with this audio and visual feedback.
The needle electrode was positioned close to a motor unit in soleus and the subject instructed to keep the unit discharging steadily while at least 250 non-random stimuli (at a maximum frequency of $1.0 \mathrm{~Hz}$ ) were delivered to either the popliteal nerve (at group I threshold) or peroneal nerve (at alpha threshold). The needle electrode was then moved to select another motor unit. The EMG signal and a pulse generated by the stimulator were recorded using a Hewlett Packard 3960 FM tape recorder.

The analysis was performed from the tape (Fig. 1). Individual motor unit action potentials were selected using a window discriminator which generated a pulse (width $3.5 \mathrm{~ms}$ ) when the signal lay within the window and simultaneous differentiation indicated that a phase reversal had occurred. The signal was then passed through an 8-16 ms delay line so that the onset of the action potential could be visualised. To determine whether the desired motor unit action potential was being consistently extracted by the window discriminator, the delayed signal was monitored on a storage oscilloscope with an expanded sweep $(2 \mathrm{~ms} / \mathrm{cm})$ triggered by the pulse from the window discriminator. A particular section of tape was replayed repeatedly until the experimenter was satisfied that the window discriminator had been adjusted to exclude all unwanted potentials or artefact on that section of tape.

The pulses from the window discriminator were

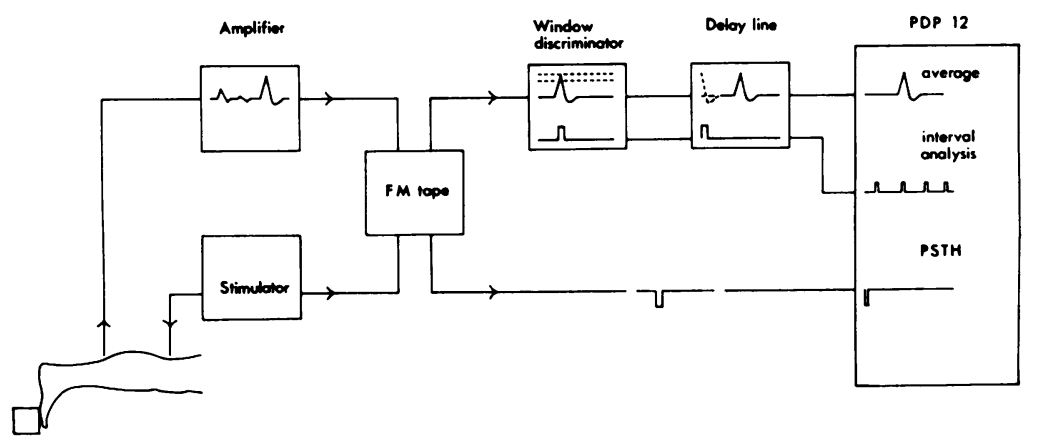

Fig. 1 Flow diagram of method. The action potentials of voluntarily activated soleus motor units are recorded with a concentric needle electrode and amplified. Individual action potentials can be selected using a window discriminator. The signal is delayed 8-16 ms so that the onset of the triggering potential can be visualised. The potential is extracted by a process of repeated averaging. Pulses from the window discriminator can be used to derive the interval and instantaneous frequency profiles, the first order interval and joint interval histograms, and the autocorrelation. Pulses from the stimulator and window discriminator can be used to generate the post-stimulus time histogram (PSTH) of the soleus motor unit discharge after stimulation of the popliteal or peroneal nerve. 
used to start the clock (set at $1000 \mathrm{~Hz}$ ) on the PDP 12 laboratory computer (Digital Equipment Corporation). At each new pulse the clock count (equivalent to the interval between motor unit discharges in milliseconds) was stored, and the clock turned back to zero. Each pulse also initiated $\mathrm{A}$ to $\mathrm{D}$ sampling (at $53 \mathrm{kHz}$ ) over a period of $27 \mathrm{~ms}$ (512 samples). A further $17 \mathrm{~ms}$ of real time was taken up with a temporary display making the turnaround time, which was the shortest interval that could be registered, $44 \mathrm{~ms}$. The EMG signal was sampled 256 or 512 times and averaged. The programme permitted the display of the averaged action potential with its peak to peak amplitude and a movable time scale, the successive intervals, the successive instantaneous frequencies (with the mean frequency), and the first order interval and joint interval histograms. The interval data, which were collected during stimulation of a peripheral nerve, represent the spontaneous interspike intervals of the neurone 'contaminated' by occasional aberrant intervals related to the stimuli.

The tape was then rerun. The clock on the PDP 12 was now triggered from the stimulator pulse. In this way the post-stimulus time histogram (PSTH) and a raster display of the discharge of the motor unit after the stimulus were generated using pulses from the window discriminator to trigger a second clock. PSTHs of $512 \mathrm{~ms}$ duration with bin widths of 1 or $4 \mathrm{~ms}$ proved most suitable. The mean height of the 512 bins was also displayed. The onset and termination of changes in impulse density were taken from crossings of the mean of the PSTH with $1 \mathrm{~ms}$ bins. The shortest interval that could be registered was $1 \mathrm{~ms}$. The autocorrelation histogram was also obtained with this programme. The computer displays were photographed from a slave oscilloscope using a Polaroid camera.

The following points should be considered during the collection and interpretation of the PSTH.

Artefacts in the PSTH If the stimulus artefact falls within the window it will be included in the average (as if it were a potential) and contribute to the first bin of PSTH. If it is so large that it occludes the window the first bin of the PSTH will be empty. If the stimulus current delivered to the popliteal nerve is too great the monosynaptic reflex discharge of several soleus motor units may occur. Should this compound action potential fall within the window it will be included in the average and will contribute to the appro- priate bin of the PSTH. Should the compound potential be even larger it will occlude the window and produce a gap in the PSTH. These artefacts can be detected only by scrupulous monitoring of the signal extracted by the window discriminator while each section of tape is replayed several times.

Measurement of latencies in the PSTH As the window discriminator generates a pulse at the peak of the motor unit action potential the rise time of that particular action potential (and in the present study, because the computer was triggered from the falling edge of the pulse, the width ( $3.5 \mathrm{~ms})$ of the generated pulse) must be subtracted from all latencies in the PSTH if they are to be compared with latencies measured to the onset of the action potential in the conventional way. To avoid confusion most PSTH histogram latencies in this study will be presented without this correction (of about $8 \mathrm{~ms}$ ) unless specifically stated.

Interpretation of the PSTH The PSTH represents the probability of threshold crossing and can, therefore, be used to infer PSPs. The way in which the profile of the PSP is reflected in the PSTH is complex and had been the subject of mathematical analysis and computer simulation (Moore et al., 1970; Bryant et al., 1973; Knox, 1974; Knox and Poppele, 1977; Knox et al., 1977). Although the relationship is unpredictable and has not been simulated for the particular circumstances of the present study, the following descriptive account is believed to provide a realistic basis for the interpretation of the data to be presented.

If an EPSP causes the membrane potential to reach threshold this will occur during the rising phase of the EPSP, not during its subsequent decline (it is assumed that the discharge of a human motoneurone resets the membrane potential, effectively erasing all memory of recent PSPs). Changes in impulse density in the PSTH will, therefore, resemble the first derivative of the rising phase of the PSP rather than the PSP directly (Knox, 1974). This relationship could become distorted if the PSP were superimposed on the very steep intraspike membrane potential trajectory of a rapidly discharging neurone. Here the duration of a peak in the PSTH would tend to provide an overestimate of the rise time of the EPSP (Knox, 1974).

An increase in impulse density resulting from the rising phase of an EPSP will be followed by a 
period of reduced impulse density in the PSTH related both to the falling phase of the EPSP and to after hyperpolarisation of the neurone. The duration of this period of reduced impulse density may be determined largely by the height of the EPSP relative to the trajectory of the interspike membrane potential. For, the larger the EPSP the sooner after a spontaneous spike can the neurone be reset, and the nearer will the duration of the subsequent period of reduced impulse density in the PSTH approach the mean interspike interval of that cell.

The amplitude of an EPSP (relative to threshold) is reflected by the integral of the initial period of increased impulse density and by the duration of the subsequent period of reduced impulse density.

An IPSP will result in a period of reduced impulse density followed by a period of increased density (Knox and Poppele, 1977). For IPSPs with rapid onset and exponential decline, the period of reduced impulse density will tend to occupy a greater proportion of the duration of the whole disturbance when the IPSP is large.

Periodicity effects The stimulus may have the effect of entraining the rhythmically firing motoneurone. If this occurs there will be periodic fluctuations of impulse density occurring at multiples of the mean interspike interval. Any longlasting changes in excitability resulting from the stimulus could be masked by such periodicity effects (Moore et al., 1970; Knox and Poppele, 1977). For this reason, in the present study, interpretations will be restricted to the changes in excitability within the mean interspike interval (that is within the first $100-250 \mathrm{~ms}$ after the stimulus).

Stimuli delivered at regular intervals may provide a better estimate of PSP contour than random stimuli (Knox, 1974) but regular stimuli could, theoretically, generate periodicity effects of their own. Such effects are probably negligible when the neurone interspike interval is variable and the intervals between stimuli are long (here twice as long as the PSTH duration).

Student's (unpaired) $t$ test, the correlation coefficient, and regression (by the method of least squares) were used for statistical analysis.

\section{Results}

CHARACTERISTICS OF SOLEUS MOTOR UNITS

All the subjects were able to maintain a stable discharge of some soleus motor units with the assistance of visual and audio feedback. However, only units recruited during a gentle to moderate contraction could be isolated (even with the window discriminator), and some subjects had difficulty in maintaining certain units within this range so the possibility of selection arises. In all, 30 motor units from seven subjects were studied but the data were incomplete for three. The amplitudes and durations of the motor unit action potentials and their mean discharge frequencies are shown in Fig. 2. At the level of the contraction used, which was similar in all subjects, the motor units with the largest action potentials tended to discharge more slowly (correlation coefficient between $(\log )$ action potential amplitude and discharge frequency $r=-0.61 ; \mathrm{P}<0.001$, Fig. 3). The interval and joint interval histogram provided a visual assessment of the variability of
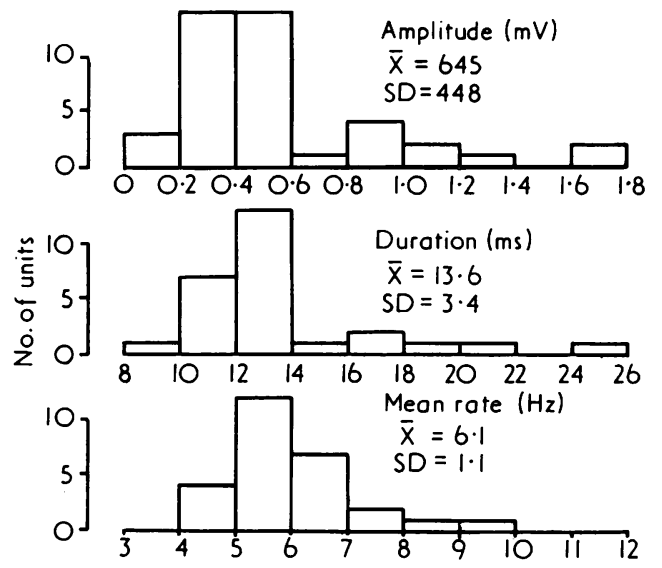

Fig. 2 Amplitudes and durations of the action potentials and the mean discharge frequencies of 27 soleus motor units.

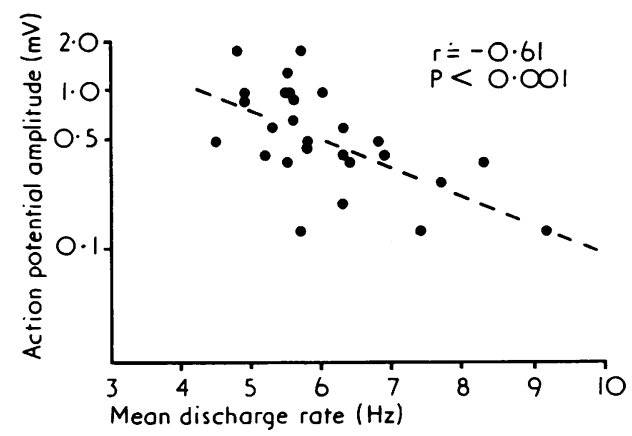

Fig. 3 Relationship between the discharge rate of a motor unit and the amplitude of the unit's action potential (note the logarithmic scale on the ordinate). 
each neurone's interspike intervals. The autocorrelation histogram provided an indication of the periodicity effects to be expected from entrainment of motoneurone discharge.

RESPONSE OF SOLEUS MOTOR UNITS TO STIMULATION OF THE POPLITEAL NERVE

In most instances, subjects were able to keep the unit discharging regularly when group I threshold stimulation of the popliteal nerve was introduced. This threshold was extremely critical. A slight decrease in the stimulus current was found to have no influence on the discharge pattern of the neurone and was probably below threshold for group I afferent fibres. A slight increase in the stimulus current often resulted in the regular monosynaptic reflex activation of a number of soleus motor units. The resulting compound action potential, which appeared at a constant latency after the stimulus, could interfere with the subsequent analysis (see Methods sectionArtefacts in the PSTH).

The discharge patterns of 28 soleus motor units were studied during group I threshold stimulation of the popliteal nerve. In 21 the stimulus produced an early period of increased impulse density in the PSTH, a later period of decreased impulse density, or both. In three the stimulus produced no alterations in impulse density in the PSTH of the motor unit being studied even though the occurrence of occasional monosynaptic reflex responses of other soleus motor units indicated that the stimulus must have been above threshold for some Ia afferent fibres. These three units could not be distinguished from the other units on the basis of their rate of discharge, or the amplitude or duration of their action potentials. In four instances the stimulus was considered to be below the threshold for group I afferent fibres as there was no alteration in the PSTH attributable to the stimulus and no reflex discharge of any motor unit, in the vicinity of the needle electrode, during the record.

\section{EARLY INCREASE IN IMPULSE DENSITY}

A peak of increased impulse density occurred early in the PSTH of 18 units. In seven of these studies, however, occasional action potentials of other soleus units, excited monosynaptically, could not be excluded by the window discriminator. Since these compound action potentials occurred at a constant latency after the stimulus, and could have contributed to an early peak in the PSTH, such records were rejected. Contamination of this sort could be excluded confidently for 11 units.
For these units the period of increased impulse density in the PSTH occurred at a mean latency of $41.5 \mathrm{~ms}$ and had a mean duration of $3.6 \mathrm{~ms}$ (Figs. 4, 5). The mean ratio of the height of this peak of impulse density to the mean level of the histogram was 24.6 to 1 . As the PSTH is derived from pulses generated at the peak of the negative spike of the motor unit action potential, the rise time of the potential (usually between 3.5 and 5.5 $\mathrm{ms}$ ) and, because of the triggering polarity used, the width of the pulse $(3.5 \mathrm{~ms})$ must be subtracted from all latencies measured on the PSTH. The corrected latencies of the early peak in the PSTH (mean $34 \mathrm{~ms}$ ) correlated well with the latencies to the onset of the maximum $\mathrm{H}$ reflex, recorded with surface electrodes in each subject $(r=0.84$; $\mathbf{P}<0.05)$ although the latencies to the PSTH peaks were always longer (mean difference $3.8 \mathrm{~ms}$ ).
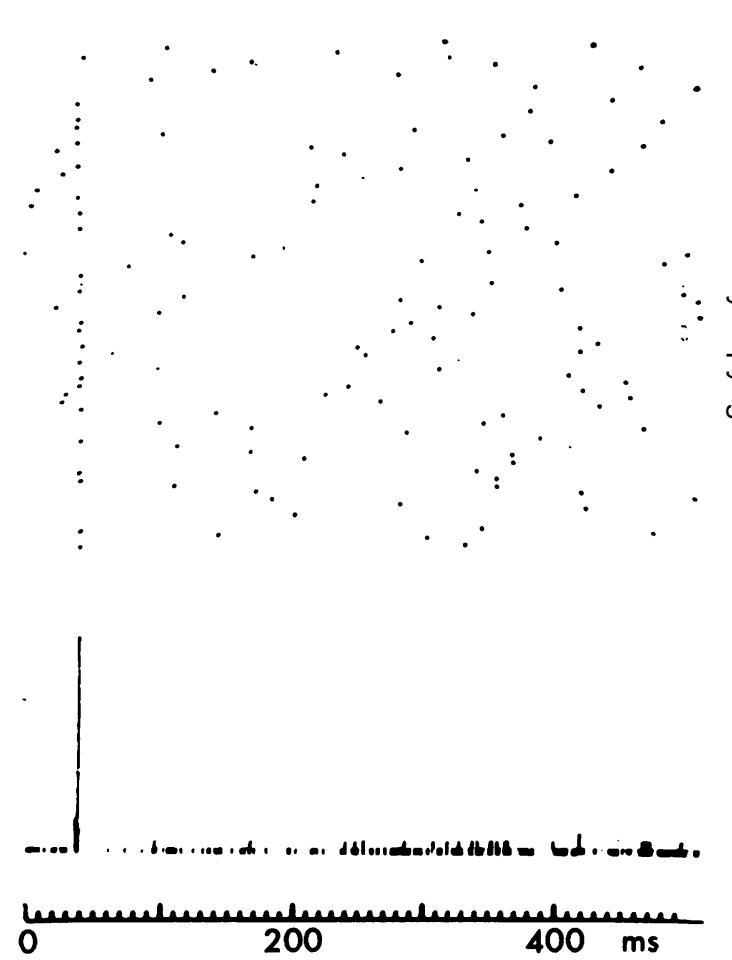

Fig. 4 Raster and PSTH of a soleus motor unit (discharging with a mean interspike interval of $217 \mathrm{~ms}$ ) after stimulation of the popliteal nerve at group $I$ threshold'. The early peak of increased impulse density has a duration above the mean level (not shown) of $2 \mathrm{~ms}$ and a latency of $40 \mathrm{~ms}$. This latency, corrected for the rise time of the action potential $(4.5 \mathrm{~ms})$ and the width of the pulse generated by the window discriminator $(3.5 \mathrm{~ms})$, is $32 \mathrm{~ms}$. This was similar to the latency of the $H$ reflex for that subject. 


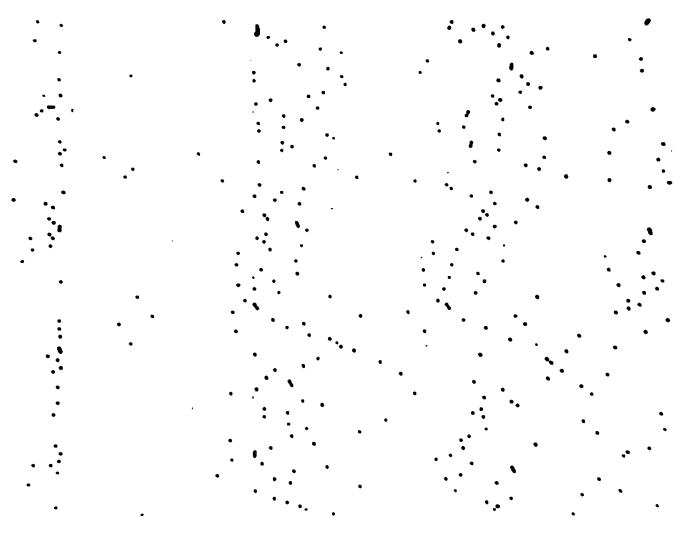

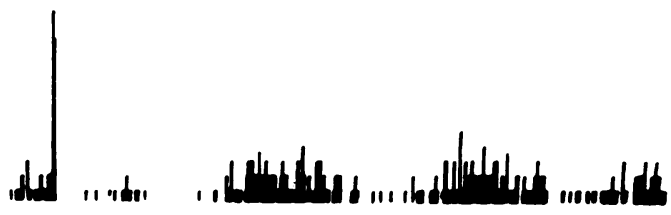

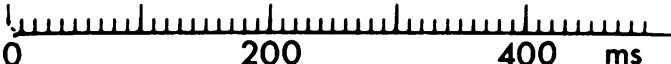

Fig. 5 Raster and PSTH of a soleus motor unit

(discharging with a mean interspike interval of $179 \mathrm{~ms}$ ) after stimulation of the popliteal nerve at 'group I threshold'. The early increase in impulse density (duration above mean $3 \mathrm{~ms}$ ), is followed by a period of reduced impulse density (to approximately $160 \mathrm{~ms}$ ) interrupted (at $90 \mathrm{~ms}$ ) by a transient increase. Periodicity effects probably account for the periods of increased impulse density with latencies of approximately $200 \mathrm{~ms}$ and $360 \mathrm{~ms}$. In this and subsequent PSTH the latencies have not been corrected for the rise time of the action potential or the width of the triggering pulse.

The amplitude of the early peak was unrelated to firing frequency. It appeared to be greater in the units with the largest action potential amplitudes (correlation coefficient between action potential amplitude and the ratio of early peak amplitude to mean PSTH amplitude: $r=0.59$; $P<0.05)$ and broader in units with action potentials of long duration (correlation coefficient between action potential duration and duration of the early increase in impulse density $r=0.77$; $\mathrm{P}<0.01$ ).

LATE REDUCTION IN IMPULSE DENSITY

Of the 24 units in which stimulation was considered to be above threshold, 21 showed a period of reduced impulse density that had its onset at a mean of $40 \mathrm{~ms}$ and its termination at a mean of $162 \mathrm{~ms}$ after the stimulus (this and all subsequent latencies are not corrected for the rise time of the action potential or the width of the triggering pulse). In 18 the termination was followed by an identifiable peak of increased impulse density at a mean latency of $226 \mathrm{~ms}$. Periodicity, as a consequence of the initial peak of increased impulse density, probably accounts for this latter peak as the interval between the two peaks was correlated with the mean interspike interval of that unit $(r=0.42 ; \mathrm{P}<0.05)$. The period of reduced impulse density occupied a mean of $69 \%$ of the mean interspike interval. In five units the period of reduced impulse density was interrupted by a transient increase in impulse density that had a mean latency of $90 \mathrm{~ms}$ (Fig. 5). In three units, all in one subject, a period of reduced impulse density (between mean latencies of 50 and $163 \mathrm{~ms}$ ) was observed in the PSTH even though there was no preceding early facilitation to produce entrainment of motoneurone firing (Fig. 6).

RESPONSE OF SOLEUS MOTOR UNITS TO STIMULATION OF THE PERONEAL NERVE

Stimulation of the peroneal nerve produced alter-

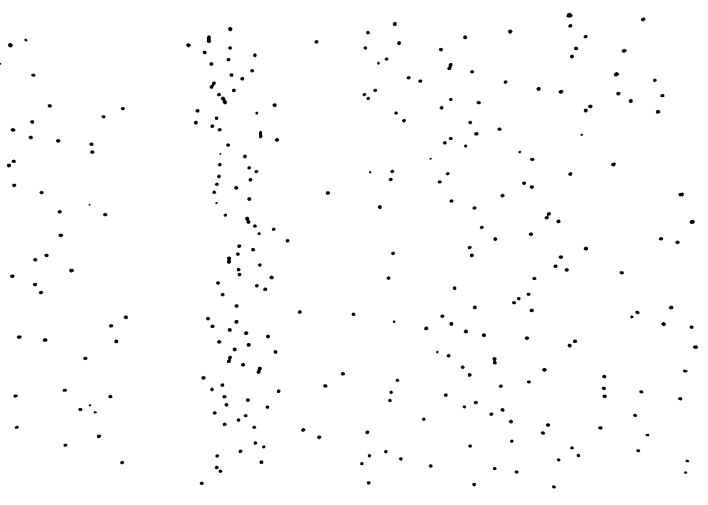

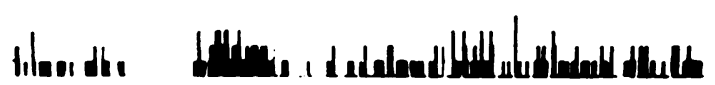

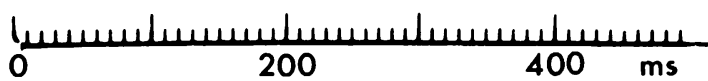

Fig. 6 Raster and PSTH of a soleus motor unit (discharging with a mean interspike interval of $175 \mathrm{~ms}$ ) after stimulation of the popliteal nerve at 'group I threshold'. There is a period of reduced impulse density from 85-136 ms after the stimulus without any preceding period of increased impulse density. 
ations in the discharge pattern of eight of the nine units examined.

A period of reduced impulse density was observed early in the PSTH of five units (Fig. 7). This began a mean of $42.8 \mathrm{~ms}$ after the stimulus and had a mean duration of $16.4 \mathrm{~ms}$. This was followed by a period of increased impulse density which also occurred in three units in the absence of the initial reduction. The peak of this increased density occurred at a mean latency of $79 \mathrm{~ms}$ after the stimulus. These latencies are not corrected for action potential rise time or the width of the triggering pulse.

\section{Discussion}

The soleus motor units examined were probably selected to some extent since only units activated by a gentle to moderate contraction could be extracted for the study (even with the assistance of the window discriminator) and since occasional units within this range could not be readily maintained. The range of action potential amplitudes is slightly smaller than the range reported by Buchthal and Rosenfalck (1973).

At the level of contraction used, which was
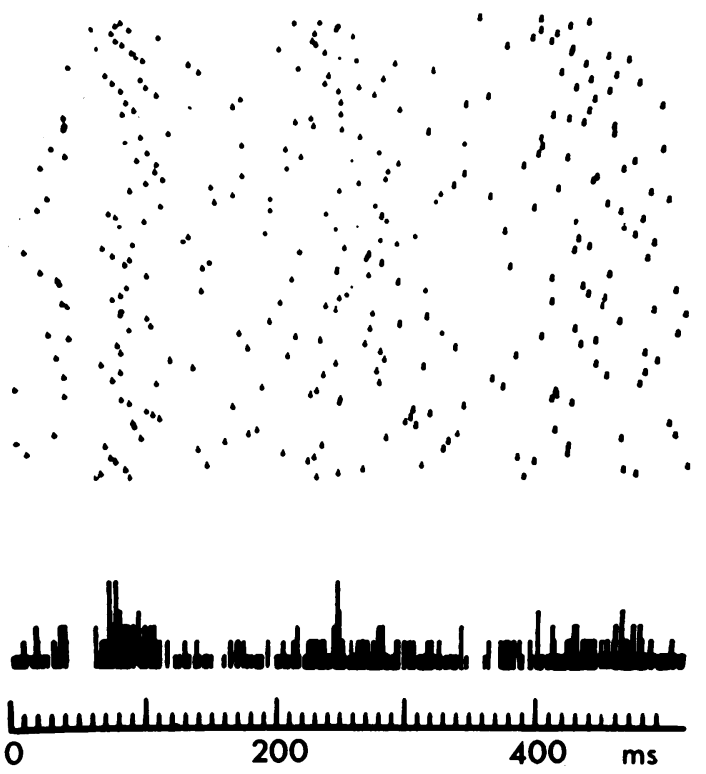

Fig. 7 Raster and PSTH of a soleus motor unit (discharging with a mean interspike interval of $192 \mathrm{~ms}$ ) after stimulation of the peroneal nerve just above threshold for the alpha motor fibres. There is a period of reduced impulse density below the mean level (not shown) between 39-61 ms after the stimulus. similar for all subjects, the units with smaller action potentials tended to discharge more rapidly. If the amplitude of a motor unit action potential is related to the size of its motoneurone (Olson et al., 1968), this finding is in agreement with more direct evidence (Milner-Brown et al., 1973; Tanji and Kato, 1973; Freund et al., 1975) indicating that motoneurones in man are recruited according to the 'size principle' (Henneman et al., $1965)$ even in relatively homogeneous muscles such as soleus (Olson et al., 1968; Buchthal and Schmalbruch, 1970a) in which $90 \%$ of the muscle fibres are said to be of type I (Buchthal and Schmalbruch, 1970b; Johnson et al., 1973).

The initial peak of increased impulse density in the PSTH of soleus motoneurones following group I threshold stimulation of the popliteal nerve probably represents the monosynaptic activation of these motoneurones. The latency (even when the rise time of the action potential is taken into account) was longer than that to the onset of the maximum $\mathrm{H}$ reflex in that individual, but this might be expected. The effective site of stimulation may depend on stimulus intensity (Wiederholt, 1970). Group I volleys near threshold may first activate smaller motoneurones whose axons have slower conduction velocities than the motoneurones activated by a maximum group $I$ volley (Buchthal and Schmalbruch, 1970a). The reflex response of single soleus motor units in man to low intensity group I volleys may be up to $2.5 \mathrm{~ms}$ later than responses to stimuli of greater intensity. Trontelj (1973) has suggested that EPSPs produced by such low intensity stimuli might have more gradual slopes.

If alterations in impulse density in the PSTH can be taken to represent the first derivative of the rising phase of an EPSP (Knox, 1974; Knox and Poppele, 1977), the duration of the initial period of increased impulse density represents the rise time of the Ia EPSP in soleus motoneurones in man. If so, it is somewhat longer $(3.6 \mathrm{~ms})$ than the rise time of the composite EPSP in extensor motoneurones in the cat which lies between 0.6 and $2.0 \mathrm{~ms}$ (Brock et al., 1952; Burke, 1968). There may be technical explanations for this difference. In the first place the resolution of the method is limited by the bin width of the histogram (here $1.0 \mathrm{~ms}$ ). Secondly, there is likely to be some 'jitter' in the exact position of the peak of the extracted action potential (especially when it is superimposed on an irregular baseline), which could slur the PSTH peak. (It was noted that the initial PSTH peak was broader in motor units with long duration action potentials in which 
'jitter' is more likely to occur.) Thirdly, alterations in impulse density in the PSTH may not represent the pure first derivative of the PSP contour if the neurone is discharging rapidly (Knox, 1974). Finally, threshold stimulation may result in composite EPSPs that have a slower rise time than when more intense stimulation is used (Trontelj, 1973). Certain of these effects might be minimised by reducing the PSTH bin width and examining only slowly discharging neurones.

If the longer rise time of the Ia EPSP in man were confirmed it might indicate that there is greater temporal dispersion of the arrival times of the individual Ia EPSPs in man, that the Ia terminals are generally more distal on the motoneurone dendrites in man, that there is greater spatial dispersion of the Ia boutons on the somatodendritic membrane, or that the dynamics of transmitter release or the membrane time constants or both are different in man.

It does not appear possible to derive the duration of the falling phase of the EPSP from the PSTH when regular stimulation is used.

Soleus motoneurones are known to receive an important Ia input in the cat (Eccles et al., 1957; Burke, 1968; Scott and Mendell, 1976). This is probably also the case in man. An early peak of increased density was observed in the PSTH of $75 \%$ of the soleus units examined. The amplitude of this initial peak was large. The subsequent period of reduced impulse density occupied almost $70 \%$ of the interspike interval of the neurone. This suggests that the composite Ia EPSP in man can be large enough to reset a rhythmically discharging neurone early in the interspike interval when the membrane potential would be furthest from threshold (Eccles, 1953; Calvin, 1975). This is not surprising since the $\mathrm{H}$ reflex is readily obtained in soleus, indicating that the Ia volley is sufficient in itself to bring even quiescent soleus motoneurones to threshold. In some of the present studies group I volleys appeared to produce an almost obligatory discharge of soleus motoneurones.

In the cat Ia EPSPs are larger and longer in duration in smaller motoneurones (Eccles et al., 1957; Burke, 1968; Burke et al., 1976); but in man; unexpectedly, the early increase in impulse density was greater in the soleus motor units with the largest action potentials. The size of the action potential, however, may not provide the most reliable indication of motor unit size. Studies of the present type, with the further characterisation of the motor unit by its twitch tension (MilnerBrown et al., 1973) or axon conduction velocity
(Freund et al., 1975) would determine whether there are any important differences between the two species.

Certain motoneurones showed no early facilitation even though a monosynaptic reflex response in other units indicated that an afferent volley was reaching the spinal cord. These neurones might have been in the upper ranks of the recruitment order (although they did not have larger action potentials). It is possible that Ia terminals may not be distributed equally to all soleus motoneurones in man.

In some instances a period of reduced impulse density was observed in the PSTH in the absence of a preceding peak to entrain the neurone. In other instances a brief period of increased impulse density (which must indicate the rising phase of an EPSP or the termination of an IPSP) appeared at a latency of $90 \mathrm{~ms}$. These late effects could arise from slowly conducting afferent fibres and/ or polysynaptic transmission including: polysynaptic effects resulting from the group I volley (Marsden et al., 1972; Lee and Tatton, 1975); effects arising from the cutaneous afferent fibres under the stimulating electrode (Hagbarth, 1960; Gassel and Ott, 1970; Caccia et al., 1973; Hugon, 1973). the presynaptic modulation (from primary afferent fibres) of background afferent barrage to motoneurones (Schmidt, 1971; Rudomin et al., 1974).

Periodicity effects complicate the interpretation of the PSTH at intervals greater than the mean interspike interval of the neurone under study. It is possible that this problem could be overcome by serial subtraction of scaled autocorrelation histograms so as to represent the entraining effects of the initial peak of the PSTH.

Stimulation of the peroneal nerve was sufficient to excite some alpha motor fibres and must, therefore, have been above the threshold of the group I afferent fibres. The initial period of reduced impulse density observed in the PSTH of soleus units after peroneal nerve stimulation may be the counterpart of the Ia IPSP. The latency is appropriate but the duration is rather too long (Brock et al., 1952; Coombs et al., 1955; Tanaka, 1972). Other inhibitory effects-for example, from the activation of cutaneous nerves under the electrode-may contribute to this period of reduced impulse density. Although antidromic activation of some alpha motor axons in the peroneal nerve must have occurred this would not be expected to give rise to Renshaw cell inhibition of the antagonist soleus motoneurones (Wilson et al., 1960; Eccles et al., 1961). The subsequent peak of in- 
creased impulse density may represent the differentiated rising phase of the Ia IPSPs but biphasic effects from the antagonist group I or cutaneous volleys cannot be excluded.

The present technique is relatively simple and could be carried out with standard electromyographic amplifiers supplemented by a window discriminator, a delay line, and a small averaging computer capable of generating a PSTH. The method appears to have several advantages.

1. It is possible to derive some of the characteristics of PSPs in single motoneurones in man.

2. The method may be capable of detecting quite small PSPs provided they have a rapid rise time (Knox and Poppele, 1977).

3. Studies are not restricted to muscle groups in which a monosynaptic reflex can be obtained.

There are also two main problems:

1. The contour of the PSP is reflected by the contour of the PSTH in a complex manner. The many variables include the rate and regularity of both the delivered stimuli and the neurone's discharge. For this reason the details of PSP shape may be most readily predicted at present by computer simulation (Knox et al., 1977).

2. Interpretation of late changes in excitability at intervals longer than the mean interspike interval are complicated by periodicity effects. These may eventually be correctable.

We conclude that the present method could be used to examine the afferent connections to spinal motoneurones in normal man in the same systematic way as this has been carried out in the cat (Eccles and Lundberg, 1958) and to determine whether such connections are altered after lesions of the nervous system in man (McCouch et al., 1958).

This research was supported by funds of the JP Bickell Foundation and the Muscular Dystrophy Association of Canada. The authors wish to express their gratitude to Dr R. E. Burke, Dr C. K. Knox, and Dr R. E. Poppele for many important suggestions during the preparation of this manuscript.

\section{References}

Bouaziz, Z., Bouaziz, M., and Hugon, M. (1975). Modulation of soleus electromyogram during electrical stimulation of medial gastrocnemius nerve in man. Electromyography and Clinical Neurophysiology, 15, 31-42.

Brock, L. G., Coombs, J. S., and Eccles, J. C. (1952). The recording of potentials from motoneurones with an intracellular electrode. Journal of Physiology, 117, 431-460.
Bryant, H. L., Marcos, A. R., and Segundo, J. P. (1973). Correlations of neuronal spike discharges produced by monosynaptic connections and by common inputs. Journal of Neurophysiology, 36, 205225.

Buchthal, F., and Rosenfalck, P. (1973). On the structure of motor units. In New Developments in Electromyography and Clinical Neurophysiology, Vol. 1, pp. 71-85. Edited by J. E. Desmedt. Karger: Basel.

Buchthal, F., and Schmalbruch, H. (1970a). Contraction times of twitches evoked by H-reflex. Acta Physiologica Scandinavica, 80, 378-382.

Buchthal, F., and Schmalbruch, H. (1970b). Contraction times and fibre types in intact human muscle. Acta Physiologica Scandinavica, 79, 435-452.

Burke, R. E. (1968). Group Ia synaptic input to fast and slow twitch motor units of cat triceps surae. Journal of Physiology, 196, 605-630.

Burke, R. E., Rymer, W. Z., and Walsh, J. V. (1976). Relative strength of synaptic input from shortlatency pathways to motor units of defined type in cat medial gastrocnemius. Journal of Neurophysiology, 39, 447-458.

Caccia, M. R., McComas, A. J., Upton, A. R. M., and Blogg, T. (1973). Cutaneous reflexes in small muscles of the hand. Journal of Neurology, Neurosurgery, and Psychiatry, 36, 960-977.

Calvin, W. H. (1975). Generation of spike trains in CNS neurons. Brain Research, 84, 1-22.

Coombs, J. S., Eccles, J. C., and Fatt, P. (1955). The inhibitory suppression of reflex discharges from motoneurones. Journal of Physiology, 130, 396-413.

Eccles, J. C. (1953). The Neurophysiological Basis of Mind. The Principles of Neurophysiology. Clarendon Press. Oxford.

Eccles, J. C., Eccles, R. M., Iggo, A., and Ito, M. (1961). Distribution of recurrent inhibition among motoneurones. Journal of Physiology, 159, 479-499.

Eccles, J. C., Eccles, R. M., and Lundberg, A. (1957). The convergence of monosynaptic excitatory afferents on to many different species of alpha motoneurones. Journal of Physiology, 137, 22-50.

Eccles, R. M., and Lundberg, A. (1958). Integrative patterns of Ia synaptic actions on motoneurones of hip and knee muscles. Journal of Physiology, 144, 271-298.

Freund, H. J., Budingen, H. J., and Dietz, V. (1975). Activity of single motor units from human forearm muscles during voluntary isometric contractions. Journal of Neurophysiology, 38, 933-946.

Gassel, M. M., and Ott, K. H. (1970). Local sign and late effects on motoneuron excitability of cutaneous stimulation in man. Brain, 93, 95-106.

Hagbarth, K. E. (1960). Spinal withdrawal reflexes in the human lower limbs. Journal of Neurology, Neurosurgery, and Psychiatry, 23, 222-227.

Henneman, E., Somjen, G., and Carpenter, D. 0. (1965). Functional significance of cell size in spinal motoneurons. Journal of Neurophysiology, 28, 560 580. 
Hugon, M. (1973). Exteroceptive reflexes to stimulation of the sural nerve in normal man. In New Developments in Electromyography and Clinical Neurophysiology. Vol. 3, pp. 713-729. Edited by J. E. Desmedt. Karger: Basel.

Johnson, M. A., Polgar, J., Weightman, D., and Appleton, D. (1973). Data on the distribution of fibre types in thirty-six human muscles. An autopsy study. Journal of the Neurological Sciences, 18, 111-129.

Knox, C. K. (1974). Cross-correlation functions for a neuronal model. Biophysical Journal, 14, 567-582.

Knox, C. K., and Poppele, R. E. (1977). Correlation analysis of stimulus evoked changes in excitability of spontaneously firing neurons. Journal of Neurophysiology. In press.

Knox, C. K., Kubota, S., and Poppele, R. E. (1977). A determination of excitability changes in DSCT neurons from spike train analysis. Journal of Neurophysiology. In press.

Lee, R. G., and Tatton, W. G. (1975). Motor responses to sudden limb displacements in primates with specific CNS lesions and in human patients with motor system disorders. Canadian Journal of Neurological Sciences, 2, 285-293.

Marsden, C. D., Merton, P. A., and Morton, H. B. (1972). Servo action in human voluntary movement. Nature, 238, 140-143.

McCouch, G. P., Austin, G. M., Liu, C. N., and Liu, C. Y. (1958). Sprouting as a cause of spasticity. Journal of Neurophysiology, 21, 205-216.

Milner-Brown, H. S., Stein, R. B., and Yemm, R. (1973). The orderly recruitment of human motor units during voluntary isometric contractions. Journal of Neurophysiology, 230, 359-370.

Moore, G. P., Segundo, J. P., Perkel, D. H., and Levitan, H. (1970). Statistical signs of synaptic interaction in neurons. Biophysical Journal, 10, 876900.
Olson, C. B., Carpenter, D. P., and Henneman, E. (1968). Orderly recruitment of muscle action potentials. Motor unit threshold and EMG amplitude. Archives of Neurology (Chicago), 19, 591-597.

Rudomin, P., Núñez, R., Madrid, J., and Burke, R. E. (1974). Primary afferent hyperpolarization and presynaptic facilitation of Ia afferent terminals induced by large cutaneous fibres. Journal of Neurophysiology, 37, 413-429.

Schmidt, R. F. (1971). Presynaptic inhibition in the vertebrate central nervous system. Ergebnisse der Physiologie, 63, 20-101.

Scott, J. G., and Mendell, L. M. (1976). Individual EPSPs produced by single triceps surae Ia afferent fibers in homonymous and heteronymous motoneurons. Journal of Neurophysiology, 39, 679-692.

Shahani, B. (1970). Flexor reflex afferent nerve fibres in man. Journal of Neurology, Neurosurgery, and Psychiatry, 33, 786-791.

Táboríková, H., and Sax, D. S. (1969). Conditioning of $\mathrm{H}$ reflexes by a preceding subthreshold $\mathrm{H}$-reflex stimulus. Brain, 92, 203-212.

Tanaka, R. (1972). Activation of reciprocal Ia inhibitory pathway during voluntary motor performance in man. Brain Research, 43, 649-652.

Tanji, J., and Kato, M. (1973). Recruitment of motor units in voluntary contraction of a finger muscle in man. Experimental Neurology, 40, 759-770.

Trontelj, J. V. (1973). A study of the H-reflex by single fibre EMG. Journal of Neurology, Neurosurgery, and Psychiatry, 36, 951-969.

Wiederholt, W. C. (1970). Stimulus intensity and site of excitation in human median nerve sensory fibres. Journal of Neurology, Neurosurgery, and Psychiatry, 33, 438-441.

Wilson, V. J., Talbot, W. H., and Diecke, P. J. (1960). Distribution of recurrent facilitation and inhibition in cat spinal cord. Journal of Neurophysiology, 23, 144-153. 